\title{
Association between Bone Mineral Density Original Association between Bone Mineral Density Article and Sleep Duration in the Korean Elderly Population
}

\author{
Narae Kim, Hyun-Rim Choi*, Sang-Won Kim, Byung-Sung Kim, \\ Chang-Won Won, Sun-Young Kim
}

Department of Family Medicine, Kyung Hee University School of Medicine, Seoul, Korea

Background: An association between sleep duration and a wide spectrum of diseases has been reported, but little is known about its relationship with bone mineral density (BMD). Previously conducted studies in Korea and abroad have reported results that are controversial. The present study sought to assess whether sleep duration can be considered an independent risk factor of osteoporosis.

Methods: We included participants over the age of 60 years with data on self-reported habitual sleep duration and BMD measured with dual X-ray absorptiometry. Comprehensive data on the study sample was obtained from the Korea National Health and Nutritional Survey performed from 2008 to 2010. Sex-stratified multiple regression analyses were conducted with adjustments for possible confounding factors.

Results: There was a significant inverse dose-dependent association between sleep duration and BMD measured at total hip, femur neck, and lumbar spine for women and total hip and femur neck for men. Sex-stratified regression analyses adjusted for age and body mass index revealed that sleep duration had a negative correlation with BMD at total hip and femoral neck for both women $(\beta=-0.0048 ; \mathrm{P}=0.0172$ for total hip, $\beta=-0.0037 ; \mathrm{P}=0.0303$ for femur neck) and men ( $\beta$ $=-0.0057 ; \mathrm{P}=0.0218$ for total hip, $\beta=-0.0057 ; \mathrm{P}=0.0143$ for femur neck). For women, the significance remained after further adjustment of confounding variables.

Conclusion: Prolonged sleep duration appears to have a significant association with lower total hip and femur neck BMD in elderly women but not in elderly men.

Keywords: Sleep Duration; Bone Density; Osteoporosis; Photon Absorptiometry; Koreans; Elderly

Received: June 3, 2013, Accepted: February 25, 2014

${ }^{*}$ Corresponding Author: Hyun-Rim Choi

Tel: +82-2-958-8700, Fax: +82-2-958-8699

E-mail:fmdrchoi@gmail.com

Korean Journal of Family Medicine

Copyright (C) 2014 The Korean Academy of Family Medicine

(a) This is an open-access article distributed under the terms of the Creative Commons Attribution Non-Commercial License (http://creativecommons.org/licenses/by-nc/3.0) which permits unrestricted noncommercial use, distribution, and reproduction in any medium, provided the original work is properly cited.

\section{INTRODUCTION}

In accordance with population aging, incidence of osteoporosis continues to increase, giving rise to a growing concern for its impact that crosses medical, social, and economic lines. ${ }^{1)}$ One in three women older than 50 years was reported to be suffering from osteoporosis. ${ }^{2)}$ Because resultant fractures are associated with an increased rate of morbidity and mortality, such a high prevalence of the disease stresses the importance of clarifying all possible risk factors for better prevention of osteoporosis. 
Aging, estrogen deficiency, low body or muscle mass, and low calcium intake are widely known as risk factors for osteoporosis. ${ }^{3)}$ Lifestyle patterns such as excessive alcohol consumption and decreased physical activity are also recognized to be closely linked with the disease. Previous studies have demonstrated an association between sleep duration and a wide spectrum of diseases; sleep deprivation was found to be associated with cardiovascular diseases and cancer, ${ }^{4,5)}$ while prolonged sleep duration, e.g., 9 hours or more per day has been reported to raise the risk of stroke by $65 \%$ compared to people with habitual sleep duration of 7 to 8 hours. ${ }^{5)}$ Unfortunately, little is known about the relationship between sleep duration and bone mineral density (BMD), and to the best of our knowledge, only a few studies have been carried out on the subject to date. Moreover, former studies conducted in Korea to elucidate their relationship had some limitations due to a lack of quantified information on the exact sleeping hours or the inability to represent the Korean general population. ${ }^{6-8)}$ Not only the controversial results shown by the previous reports but also the ultimate lack of studies build an unclear relationship between sleep duration and BMD.

Therefore, we performed a large-scale study to determine whether sleep duration can be considered an independent risk factor for osteoporosis in the elderly population by using data provided by the Korea National Health and Nutritional Survey (KNHANES) with quantified information on sleep duration to wholly represent the Korean general population.

\section{METHODS}

\section{Study Subjects}

Data used in the analysis were obtained from the KNHANES performed between 2008 and 2010. KNHANES is a nationwide, extensive survey performed annually in South Korea by the Korea Center for Disease Control and Prevention. Purpose of the survey is to assess health and nutritional status of adults and children in Korea and to provide health statistics that properly represent the nation. It consists of a health interview survey, a nutrition survey, and a medical examination survey. Written informed consent was obtained from all participants.

Participation rate in the survey in 2008, 2009, and 2010 was $77.8 \%, 82.8 \%$, and $81.9 \%$ of the target population, respectively.
Of the 21,811 adult participants, we only included those older than 60 years of age. Participants were excluded if data on sleeping hours or BMD measurements were missing. Other exclusion criteria were immobilization, premature ovarian failure, surgical menopause, current fracture, thyroidal disease, renal failure, or rheumatoid arthritis, leaving 2,679 for analysis.

\section{Variable Definitions and Measurements}

Information on habitual sleep duration was obtained by a selfadministered questionnaire by asking "how many hours do you sleep a day on average?" in an open-ended fashion. Participants were categorized into the following quartiles based on average sleep duration: $<6$ hours, 6 to $<7$ hours, 7 to $<8$ hours, and $\geq 8$ hours. The group with the lowest quartile of average sleep duration was defined as having short sleep duration and those with the highest quartile as having prolonged sleep duration.

Bone density was measured at major scan regions, including lumbar spine (L1-4), total hip, and femoral neck, with dualenergy X-ray absorptiometry and expressed as BMD $\left(\mathrm{g} / \mathrm{cm}^{2}\right)$ and T-score, the number of standard deviations from the mean of healthy young adults with peak bone mass. Osteoporosis was defined as a BMD values falling 2.5 standard deviations below the average according to the World Health Organization criteria for diagnosing osteoporosis.

The survey was inclusive of self-reported information on socioeconomic data, lifestyle and nutritional behaviors, and medical history, as well as baseline measurements of body compositions and serum vitamin D levels. Education level was categorized into less than college and college or higher. Excessive alcohol consumption was defined as drinking 30 grams or more of pure alcohol per day. Smoking status was categorized into current smokers and never or ex-smokers. Subjects performing vigorous exercise for more than 20 minutes at least three times a week or moderate exercise or walking for more than 30 minutes at least five times a week were considered regular exercisers. Body mass index (BMI) was defined as the body weight in kilograms divided by the square of the height in meters. Vitamin $\mathrm{D}$ deficiency was considered to exist when the concentration of serum 25-hydroxy-vitamin $\mathrm{D}$ was $12 \mathrm{ng} / \mathrm{mL}$ or less, according to the criteria proposed by the Institute of Medicine. For measurement of serum vitamin $\mathrm{D}$ levels, venous blood samples were drawn and analyzed by radioimmunoassay. 


\section{Statistical Analysis}

Statistical analyses were performed after applying weighted values to the raw data of the KNHANES to reflect nationally representative prevalence estimates. All calculations were performed separately for men and women. Baseline characteristics of the study participants were compared by using a student t-test for continuous variables and chi-square test for categorical variables, presented as mean \pm standard error or as percentages, respectively.

Multivariable-adjusted linear regression models were used to

Table 1. Baseline characteristics of study subjects*

\begin{tabular}{|c|c|c|c|}
\hline Characteristic & Male $(\mathrm{n}=1,405)$ & Female $(\mathrm{n}=1,274)$ & P-value \\
\hline Age (y) & $68 \pm 0.2$ & $69.1 \pm 0.2$ & 0.0002 \\
\hline Education (college or higher) & $37.5(1.8)$ & $12.5(1.1)$ & $<0.0001$ \\
\hline \multicolumn{4}{|l|}{ Body composition } \\
\hline Height (cm) & $165.7 \pm 0.2$ & $151.9 \pm 0.2$ & $<0.0001$ \\
\hline Weight (kg) & $64.7 \pm 0.3$ & $55.9 \pm 0.3$ & $<0.0001$ \\
\hline Body mass index $\left(\mathrm{kg} / \mathrm{m}^{2}\right)$ & $23.5 \pm 0.1$ & $24.2 \pm 0.1$ & $<0.0001$ \\
\hline Waist circumference (cm) & $85.3 \pm 0.3$ & $83 \pm 0.3$ & $<0.0001$ \\
\hline \multicolumn{4}{|l|}{ Social behaviors } \\
\hline Regular exercise $^{\dagger}$ & $25.4(1.5)$ & $18.3(1.5)$ & $<0.0001$ \\
\hline Current smoker & $28(1.5)$ & $4.6(0.7)$ & $<0.0001$ \\
\hline Heavy drinker & $26(1.4)$ & $1.2(0.3)$ & $<0.0001$ \\
\hline \multicolumn{4}{|l|}{ Diet } \\
\hline Daily calorie intake (kcal) & $2,011 \pm 25.3$ & $1,473.2 \pm 19.0$ & $<0.0001$ \\
\hline Daily protein intake (\%) & & & 0.0002 \\
\hline$<10$ & $11.4(1.2)$ & $17.9(1.3)$ & \\
\hline $10-20$ & $82.3(1.4)$ & $77.4(1.4)$ & \\
\hline$>20$ & $6.3(0.8)$ & $4.7(0.7)$ & \\
\hline Daily calcium consumption (g) & $525.7 \pm 11.2$ & $408 \pm 19.4$ & $<0.0001$ \\
\hline Serum vitamin D level (ng/mL) & & & $<0.0001$ \\
\hline$<12$ & $8.6(1.0)$ & $17.5(1.5)$ & \\
\hline $12-20$ & $35.9(1.7)$ & $43.0(2.0)$ & \\
\hline$>20$ & $55.6(1.9)$ & $39.5(2.0)$ & \\
\hline \multicolumn{4}{|l|}{ Comorbidity (\%) } \\
\hline Hypertension & $57.1(1.6)$ & $58.9(1.6)$ & 0.4198 \\
\hline Diabetes mellitus & $20.8(1.4)$ & $18(1.4)$ & 0.1589 \\
\hline History of myocardial infarction & $2.6(0.5)$ & $0.9(0.3)$ & 0.0038 \\
\hline History of cerebrovascular attack & $2.5(0.5)$ & $1.2(0.3)$ & 0.0275 \\
\hline Family history of osteoporosis (\%) & $7.0(0.8)$ & $11.8(1.0)$ & 0.0002 \\
\hline Sleep duration (h) & $6.8 \pm 0.1$ & $6.5 \pm 0.1$ & $<0.0001$ \\
\hline
\end{tabular}

*Data are presented with means \pm standard errors for continuous variable and weighted percentages (standard errors) for categorical variables. ${ }^{\dagger}$ Vigorous exercise for more than 20 minutes at least three times a week or moderate exercise or walking for more than 30 minutes at least five times a week. 
evaluate the cross-sectional association between sleep duration and BMD. We designed three different sex-stratified regression models; model 1 was adjusted for age and BMI; model 2 was further adjusted for lifestyle behaviors such as drinking, smoking, physical activity, and coffee consumption; and model 3 was additionally adjusted for education, serum vitamin D level, calcium consumption, and comorbidities such as hypertension or diabetes mellitus. Statistical analyses were performed with SAS ver. 9.2 (SAS Institute Inc., Cary, NC, USA), and P $<0.05$ was considered significant.

\section{RESULTS}

\section{Baseline Characteristics of Study Subjects}

Table 1 summarizes the gender-specific baseline characteristics of the study participants. Among the 21,811 participants, a total of 2,679 ambulatory subjects (1,405 males and 1,274 females), meeting the inclusion criteria were included in the analysis.
Anthropometric measurements such as BMI, waist circumference, height, and weight were significantly greater in compared to women men $(\mathrm{P}<0.0001)$. Men reported an average of $6.8 \pm 0.1$ hours of sleep, while women reported sleeping $6.5 \pm 0.1$ hours per day on average men $(\mathrm{P}<0.0001)$. Females tended to be older than male participants $(\mathrm{P}=0.0002)$. Men had a significantly higher proportion of current smokers $(\mathrm{P}<0.0001)$ and heavy drinkers $(\mathrm{P}<0.0001)$, and were more likely to perform regular exercise $(P<0.0001)$ than women. In terms of comorbidities, there was no gender difference in the prevalence of hypertension ( $P=0.4198)$, diabetes mellitus $(P=0.1589)$, or liver cirrhosis ( $P$ $=0.1874)$, while dyslipidemia was more prevalent in women $(P$ $=0.0079)$, and history of myocardial infarction $(P=0.0038)$ or stroke $(\mathrm{P}=0.0275)$ was more common in men. A significantly higher proportion of women reported having a family history of osteoporosis $(\mathrm{P}=0.0002)$ and were found to be suffering from vitamin $\mathrm{D}$ deficiency $(\mathrm{P}<0.0001)$. Men were more likely to consume a higher amount of calcium $(\mathrm{P}<0.0001)$ and an appropriate amount of protein $(\mathrm{P}<0.0002)$ than women.

Table 2. Total hip, femur neck, and lumbar spine BMD by quartiles of sleep duration by gender

\begin{tabular}{|c|c|c|c|}
\hline \multirow{2}{*}{ Variable } & \multicolumn{3}{|c|}{$\mathrm{BMD}\left(\mathrm{g} / \mathrm{cm}^{2}\right)$} \\
\hline & Total hip & Femur neck & Lumbar spine \\
\hline \multicolumn{4}{|c|}{ Female $(n=1,274)$} \\
\hline \multicolumn{4}{|c|}{ Sleep duration (h) } \\
\hline$\leq 5(\mathrm{n}=327)$ & $0.745 \pm 0.006305$ & $0.5905 \pm 0.005279$ & $0.7779 \pm 0.008316$ \\
\hline $6(\mathrm{n}=302)$ & $0.7363 \pm 0.006002$ & $0.5848 \pm 0.005317$ & $0.7643 \pm 0.007114$ \\
\hline $7(\mathrm{n}=325)$ & $0.7357 \pm 0.005937$ & $0.5846 \pm 0.005394$ & $0.7639 \pm 0.007538$ \\
\hline$\geq 8(\mathrm{n}=320)$ & $0.7231 \pm 0.005947$ & $0.5756 \pm 0.005153$ & $0.7537 \pm 0.007861$ \\
\hline P for trend* & 0.0104 & 0.0467 & 0.0442 \\
\hline \multicolumn{4}{|l|}{ Male $(\mathrm{n}=1,405)$} \\
\hline \multicolumn{4}{|c|}{ Sleep duration (h) } \\
\hline$\leq 5(\mathrm{n}=253)$ & $0.9198 \pm 0.007888$ & $0.7363 \pm 0.007929$ & $0.9554 \pm 0.01041$ \\
\hline $6(\mathrm{n}=321)$ & $0.9051 \pm 0.006907$ & $0.7209 \pm 0.00649$ & $0.9431 \pm 0.009164$ \\
\hline $7(\mathrm{n}=366)$ & $0.9088 \pm 0.007168$ & $0.7381 \pm 0.007145$ & $0.9396 \pm 0.009145$ \\
\hline$\geq 8(\mathrm{n}=465)$ & $0.8958 \pm 0.006143$ & $0.7122 \pm 0.005648$ & $0.9382 \pm 0.007839$ \\
\hline P for trend* & 0.0234 & 0.0205 & 0.2564 \\
\hline
\end{tabular}

Values are presented as mean \pm standard error.

BMD: bone mineral density.

*Data were calculated by simple linear regression analysis. 


\section{Association of Sleep Duration with Bone Mineral Density and T-Score}

Table 2 shows the association of sleep duration with total hip, femur neck, and lumbar spine BMD according to sex, where each gender was segmented into four groups by sleep duration. In women, there was a significant inverse dose-dependent association between sleep duration and BMD measured at total hip, femur neck, and lumbar spine. Those with habitual sleep duration in the first quartile had a mean BMD of 0.745, 0.5905, and $0.7779 \mathrm{gm} / \mathrm{cm}^{2}$ at total hip, femur neck, and lumbar spine, respectively, compared to $0.7231,0.5756$, and $0.7537 \mathrm{gm} / \mathrm{cm}^{2}$ in those with sleep duration in the fourth quartile ( $\mathrm{P}$ for trend $=0.0104,0.0467$, and 0.0442). There was also a significant inverse dose-dependent association between BMD and sleep duration but only for BMD measured at total hip and femur neck among male participants. Men with average sleep hours in the first quartile had a mean BMD of $0.9198 \mathrm{gm} / \mathrm{cm}^{2}$ and 0.7363 $\mathrm{gm} / \mathrm{cm}^{2}$ at total hip and femur neck, respectively, while those with sleep duration in the fourth quartile had a mean BMD of $0.8958 \mathrm{gm} / \mathrm{cm}^{2}$ and $0.7122 \mathrm{gm} / \mathrm{cm}^{2}$, respectively ( $P$ for trend $=0.0234,0.0205)$. Figure 1 delineates the association between sleep duration and T-score at total hip, femur neck, and spine by gender.
3. Multiple-Linear Regression Analysis of the Association of Sleep Duration with BMD and T-score

Table 3 shows sex-stratified regression analyses adjusted for confounding variables. Those adjusted for age and BMI revealed that sleep duration had a negative correlation with $\mathrm{BMD}$ and T-score at major weight-bearing sites such as total hip and femoral neck for both women $(\beta=-0.004845 ; \mathrm{P}=0.0172$ for total hip, $\beta=-0.003663 ; \mathrm{P}=0.0303$ for femur neck) and men ( $\beta=-0.005656 ; \mathrm{P}=0.0218$ for total hip, $\beta=-0.005668 ; \mathrm{P}=$ 0.0143 for femur neck). For men, the initial significance for total hip BMD disappeared in model 2 after adjustment for social behaviors, and the significance for femur neck BMD disappeared after adjustment for additional covariates including education, comorbidities, serum vitamin $\mathrm{D}$ level, and calcium consumption in model 3. For women, on the other hand, the significance remained through models 2 and 3 .

\section{DISCUSSION}

The aim of the study was to investigate whether sleep duration is a strong risk factor of osteoporosis. In this cross-sectional study of the Korean elderly population, we observed a significant linear association between sleep duration and decreased BMD. Those with the highest quartile of sleep duration were found to have

B

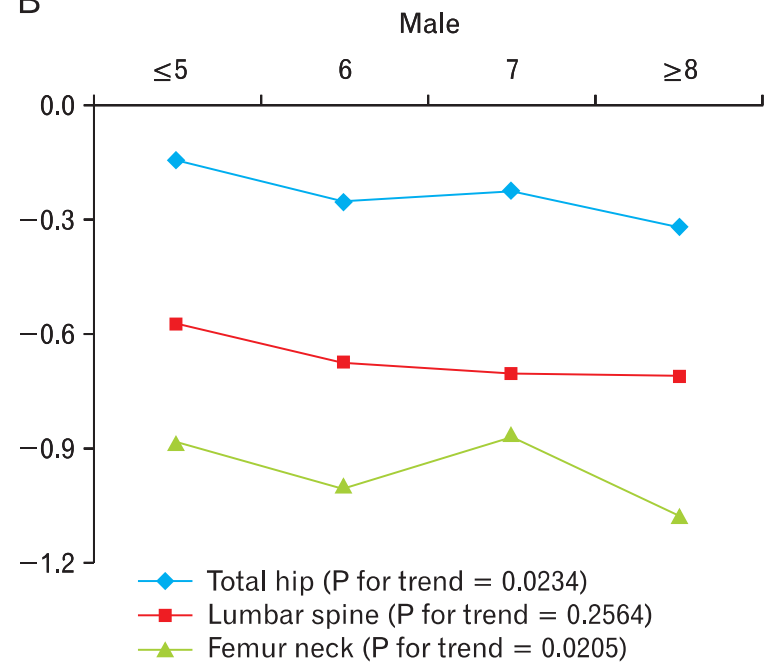

Figure 1. Total hip, femur neck, and lumbar spine T-score by quartiles of sleep duration in (A) female and (B) male. *P-values over the quartiles of sleep duration were assessed with a test for linear trends using linear regression analysis. 
Table 3. Multi-variable adjusted analysis for the association between sleep duration on BMD and T-score stratified by gender*

\begin{tabular}{|c|c|c|c|c|c|c|}
\hline \multirow{2}{*}{ Variable } & \multicolumn{2}{|c|}{ Model $1^{\dagger}$} & \multicolumn{2}{|c|}{ Model $2^{\ddagger}$} & \multicolumn{2}{|c|}{ Model $3^{\S}$} \\
\hline & $\beta$ & $\mathrm{P}$-value & $\beta$ & $\mathrm{P}$-value & $\beta$ & P-value \\
\hline \multicolumn{7}{|l|}{ Male } \\
\hline \multicolumn{7}{|l|}{$\mathrm{BMD}\left(\mathrm{g} / \mathrm{cm}^{2}\right)$} \\
\hline Total hip & -0.005656 & 0.0218 & -0.003332 & 0.1187 & -0.002985 & 0.1591 \\
\hline Femur neck & -0.005668 & 0.0143 & -0.004025 & 0.0471 & -0.003641 & 0.0611 \\
\hline Lumbar spine & -0.002722 & 0.3771 & -0.003085 & 0.3312 & -0.002373 & 0.4483 \\
\hline \multicolumn{7}{|l|}{ T-score } \\
\hline Total hip & -0.041286 & 0.0218 & -0.024323 & 0.1187 & -0.021791 & 0.1591 \\
\hline Femur neck & -0.045705 & 0.0143 & -0.032458 & 0.0471 & -0.02936 & 0.0611 \\
\hline Lumbar spine & -0.022686 & 0.3771 & -0.025709 & 0.3312 & -0.019777 & 0.4483 \\
\hline \multicolumn{7}{|l|}{ Female } \\
\hline \multicolumn{7}{|l|}{$\mathrm{BMD}\left(\mathrm{g} / \mathrm{cm}^{2}\right)$} \\
\hline Total hip & -0.004845 & 0.0172 & -0.00584 & 0.0095 & -0.00708 & 0.0031 \\
\hline Femur neck & -0.003663 & 0.0303 & -0.003945 & 0.0351 & -0.004702 & 0.017 \\
\hline Lumbar spine & -0.004372 & 0.1157 & -0.004955 & 0.1087 & -0.005711 & 0.0715 \\
\hline \multicolumn{7}{|l|}{ T-score } \\
\hline Total hip & -0.042130 & 0.0172 & -0.050783 & 0.0095 & -0.061564 & 0.0031 \\
\hline Femur neck & -0.034236 & 0.0303 & -0.036866 & 0.0351 & -0.04394 & 0.017 \\
\hline Lumbar spine & -0.038019 & 0.1157 & -0.043084 & 0.1087 & -0.049657 & 0.0715 \\
\hline
\end{tabular}

BMD: bone mineral density.

*Standardized regression coefficients of total hip, femur neck, and lumbar spine BMD with sleep duration were calculated by multiple linear regression analysis after adjusting for confounding factors. ${ }^{\dagger}$ Model 1: age and body mass index. ${ }^{\ddagger}$ Model 2: age, body mass index, smoking status, drinking status, exercise habits, and coffee consumption. ${ }^{\circledR}$ Model 3: age, body mass index, smoking status, drinking status, exercise habits, and coffee consumption, education, hypertension, diabetes mellitus, calcium intake, and serum vitamin D level.

significantly lower total hip and femur neck BMD after adjustment for age and BMI for both sexes; the significance remained after additional adjustment for all possible associated variables for females, but not for males. Additional analyses of sleep duration demonstrated that men with prolonged sleep duration were found to be suffering from diabetes mellitus in a higher proportion ( $\mathrm{P}$ $=0.0378)$ and also drink more coffee $(\mathrm{P}=0.0363)$ than shorter sleepers, possibly explaining the reason why the significance between sleep duration and BMD was not maintained after adjusting for additional confounding factors in men.

Only a few attempts had been made to elucidate the exact association between sleep duration and $\mathrm{BMD}$, and results of the few reports were unfortunately contradictory to each other. One cross-sectional study on Chinese women, showed a significant association between decreased sleep duration and lower BMD, possibly owing to hormonal factors related to sleep deprivation. ${ }^{9)}$ However, one other cross-sectional study on Japanese middleaged and elderly population showed entirely different results, ${ }^{10)}$ which is consistent with the results of the present study.

Previous studies have suggested some possible mechanisms through which prolonged sleep duration may adversely affect $\mathrm{BMD}$. According to the previously mentioned cross-sectional study on Japanese middle-aged and elderly population, hypothetically due to lessened daily mechanical loading from inactivity and lower level of light-induced estrogen levels from decreased light exposure, those reporting to sleep a longer period of time appeared to have higher odds of developing osteoporosis compared to shorter sleepers. ${ }^{10)}$ Excessive amount of sleep can 
indeed bring reductions in biomechanical forces from decreased activity or sarcopenia, which would signal less need for more bone mass, ultimately leading to elimination of bone. ${ }^{11)}$

In the present study, separate analyses by gender were necessary because baseline characteristics of the study participants, especially the anthropometric components, were observed to greatly differ according to sex, which also explains why women are more vulnerable to osteoporosis than men of the same age and body compositions. A study on infants also revealed significantly greater BMD in male infants compared with female infants, ${ }^{12)}$ and another study found that the rate of age-related decrease in femoral neck $\mathrm{BMD}$ was approximately twice as fast in women than men, who experience a more gradual decrease as they age. ${ }^{13)}$

For both sexes, prolonged sleep duration did not have an inverse association with $\mathrm{BMD}$ measured at the lumbar spine. Several structural changes within the spine are known to affect lumbar spine BMD measurements. Undiagnosed vertebral fractures or degenerative changes of the spine, such as osteophytes and facet sclerosis, have been reported to result in significantly increased lumbar spine BMD. In the presence of osteophytes, BMD was increased by $9.5 \%$ at $\mathrm{L}_{4}$ and $13.9 \%$ at $\mathrm{L}_{1}{ }^{14)}$ Subjects included in this study with undiagnosed vertebral fractures or degenerative changes of the spine may have contributed to falsely increased measurements, obscuring the genuine relationship between sleep duration and lumbar spine BMD.

To the best of our knowledge, the present study is the first to incorporate sex-specific regression models, taking into consideration that gender differences exist in sleep duration and baseline body compositions that are closely linked with BMD. By obtaining a large population-based sample from the KNHANES, we were also able to provide results that are generalizable to the Korean elderly population and to collect comprehensive data, allowing adjustment for a large number of potential confounders.

Some of the limitations to our study should also be noted. First, this study was limited to a cross-sectional design, thus temporal relationship or causality could not be established. Secondly, we used self-reported sleep duration, possibly leading to biased estimates. Studies using objectively confirmed information on sleep duration as well as sleep quality may provide more robust evidence, concerning the effect of sleep duration on BMD.

In conclusion, sleep duration holds an inverse dose-dependent association with $\mathrm{BMD}$ at total hip and femur neck among elderly women in a large representative sample of the Korean general population. Further investigations should focus on objective measurements of sleep duration.

\section{CONFLICT OF INTEREST}

No potential conflict of interest relevant to this article was reported.

\section{REFERENCES}

1. Watts NB, Bilezikian JP, Camacho PM, Greenspan SL, Harris ST, Hodgson SF, et al. American Association of Clinical Endocrinologists Medical Guidelines for Clinical Practice for the diagnosis and treatment of postmenopausal osteoporosis. Endocr Pract 2010;16 Suppl 3:1-37.

2. Ministry for Health, Welfare and Family Affairs; Korea Center for Disease Control and Prevention. The 4th Korea National Health and Nutrition Examination Survey. Cheongwon: Korea Center for Disease Control and Prevention; 2009.

3. Lane NE. Epidemiology, etiology, and diagnosis of osteoporosis. Am J Obstet Gynecol 2006;194(2 Suppl):S3-11.

4. Hoevenaar-Blom MP, Spijkerman AM, Kromhout D, van den Berg JF, Verschuren WM. Sleep duration and sleep quality in relation to 12 -year cardiovascular disease incidence: the MORGEN study. Sleep 2011;34:1487-92.

5. Von Ruesten A, Weikert C, Fietze I, Boeing H. Association of sleep duration with chronic diseases in the European Prospective Investigation into Cancer and Nutrition (EPIC)Potsdam study. PLoS One 2012;7:e30972.

6. Lee BY. A study on the factors which are related to bone mineral density of female [dissertation]. Wanju: Woosuk University; 2008.

7. Lee JH, Lee BK, Kim YS. The relationship between bone mineral density and body composition, life styles of female college students. Korean J Food Nut 2011;24:786-96.

8. Sung CJ, Choi SH, Kim MH, Choi YH, Lee DH, Baek SK, et al. A Study on nutritional status, maternal factors, and lifestyles according to BMD in rural postmenopausal women. Korean J Community Nutr 2001;6:192-204. 
9. Fu X, Zhao X, Lu H, Jiang F, Ma X, Zhu S. Association between sleep duration and bone mineral density in Chinese women. Bone 2011;49:1062-6.

10. Kobayashi D, Takahashi O, Deshpande GA, Shimbo T, Fukui T. Association between osteoporosis and sleep duration in healthy middle-aged and elderly adults: a large-scale, crosssectional study in Japan. Sleep Breath 2012;16:579-83.

11. Riggs BL, Melton LJ 3rd. The worldwide problem of osteoporosis: insights afforded by epidemiology. Bone 1995; 17(5 Suppl):505S-511S.

12. Rupich RC, Specker BL, Lieuw-A-Fa M, Ho M. Gender and race differences in bone mass during infancy. Calcif Tissue Int 1996;58:395-7.

13. Burger H, van Daele PL, Algra D, van den Ouweland FA, Grobbee DE, Hofman A, et al. The association between age and bone mineral density in men and women aged 55 years and over: the Rotterdam Study. Bone Miner 1994;25:1-13.

14. Rand T, Seidl G, Kainberger F, Resch A, Hittmair K, Schneider B, et al. Impact of spinal degenerative changes on the evaluation of bone mineral density with dual energy X-ray absorptiometry (DXA). Calcif Tissue Int 1997;60:430-3. 